\title{
APOE4-mediated amyloid- $\beta$ pathology depends on its neuronal receptor LRP1
}

\author{
Masaya Tachibana, ${ }^{1,2}$ Marie-Louise Holm, ${ }^{1,3}$ Chia-Chen Liu, ${ }^{1}$ Mitsuru Shinohara, ${ }^{1,4}$ Tomonori Aikawa, ${ }^{1}$ Hiroshi Oue, ${ }^{1}$ Yu Yamazaki, \\ Yuka A. Martens, ${ }^{1}$ Melissa E. Murray, ${ }^{1}$ Patrick M. Sullivan, ${ }^{5}$ Kathrin Weyer, ${ }^{3}$ Simon Glerup, ${ }^{3}$ Dennis W. Dickson, ${ }^{1}$ Guojun Bu, ${ }^{1}$ \\ and Takahisa Kanekiyo'
}

'Department of Neuroscience, Mayo Clinic, Jacksonville, Florida, USA. ${ }^{2}$ United Graduate School of Child Development, Osaka University, Osaka, Japan. ${ }^{3}$ Department of Biomedicine, Aarhus University, Aarhus, Denmark. ${ }^{4}$ Department of Aging Neurobiology, National Center for Geriatrics and Gerontology, Aichi, Japan. ${ }^{5}$ Department of Medicine, Duke University School of Medicine, Durham, North Carolina, USA

\begin{abstract}
Carrying the 84 allele of the APOE gene encoding apolipoprotein E (APOE4) markedly increases the risk for late-onset Alzheimer's disease (AD), in which APOE4 exacerbates the brain accumulation and subsequent deposition of amyloid- $\beta$ $(A \beta)$ peptides. While the LDL receptor-related protein 1 (LRP1) is a major apoE receptor in the brain, we found that its levels are associated with those of insoluble $A \beta$ depending on APOE genotype status in postmortem AD brains. Thus, to determine the functional interaction of apoE4 and LRP1 in brain A $\beta$ metabolism, we crossed neuronal LRP1-knockout mice with amyloid model APP/PS1 mice and APOE3-targeted replacement (APO3-TR) or APOE4-TR mice. Consistent with previous findings, mice expressing apoE4 had increased $A \beta$ deposition and insoluble amounts of $A \beta 40$ and $A \beta 42$ in the hippocampus of APP/PS1 mice compared with those expressing apoE3. Intriguingly, such effects were reversed in the absence of neuronal LRP1. Neuronal LRP1 deficiency also increased detergent-soluble apoE4 levels, which may contribute to the inhibition of $A \beta$ deposition. Together, our results suggest that apoE4 exacerbates $A \beta$ pathology through a mechanism that depends on neuronal LRP1. A better understanding of apoE isoform-specific interaction with their metabolic receptor LRP1 on A metabolism is crucial for defining APOE4-related risk for AD.
\end{abstract}

\section{Introduction}

Dementia is a devastating disorder in the aging population, progressively compromising cognitive performance. Alzheimer's disease (AD) is responsible for the development of $60 \%$ to $80 \%$ of all dementia cases (1). AD is histologically diagnosed by the presence of senile plaques and neurofibrillary tangles $(2,3)$, while neuropathological studies have revealed that pathologic features of other dementias also often coexist in postmortem AD brains (1). Although the pathogenesis of $\mathrm{AD}$ has not been fully defined, accumulating evidence supports the amyloid cascade hypothesis (4): accelerated generation of amyloid- $\beta(A \beta)$ peptides and/or disturbed $A \beta$ clearance predominantly contributes to the pathogenic pathways of the disease by triggering amyloid pathology in the brain.

Most cases of $\mathrm{AD}$ are sporadic and late onset (1), in which the $\varepsilon 4$ allele of the apolipoprotein E (APOE4) gene is the strongest genetic risk factor among its 3 polymorphic alleles $(\varepsilon 2, \varepsilon 3$, and $\varepsilon 4)$ $(5,6)$. Importantly, brain $A \beta$ pathology is inarguably associated with APOE4 status (7). While APOE4 decreases $\mathrm{A} \beta$ clearance without affecting $A \beta$ production (8), $A \beta$ pathology is substantially aggravated in amyloid mouse models when APOE 4 is overexpressed in the brain

\section{Related Commentary: p. 969}

Authorship note: MT and MLH contributed equally to this work. Conflict of interest: The authors have declared that no conflict of interest exists. License: Copyright 2019, American Society for Clinical Investigation. Submitted: September 11, 2018; Accepted: December 18, 2018 Reference information: / Clin Invest. 2019;129(3):1272-1277. https://doi.org/10.1172/JCl124853. during the seeding stage of amyloid development (9). Thus, the importance of exploring APOE4-related $\mathrm{A} \beta$ metabolism has been increasingly recognized for dissecting the complex pathogenesis of $\mathrm{AD}$. The major role of apoE isoforms, mainly produced by astrocytes in the brain, is to deliver cholesterol and other lipids to neurons via cell-surface receptors, such as the LDL receptor family and heparan sulfate proteoglycans (HSPGs) $(5,10)$. A major apoE receptor in the brain is LDL receptor-related protein 1 (LRP1), which is abundantly expressed in a variety of brain cell types in particular neurons. LRP1 has the ability to endocytose an array of ligands, including apoE and $A \beta$, in a highly efficient manner, by coupling to other transmembrane proteins $(5,10)$. However, it remains unknown how LRP1 modulates APOE4-mediated A $\beta$ metabolism in AD.

Therefore, we investigated the contributions of APOE4 and LRP1 to A $\beta$ pathology using postmortem brain tissues from $\mathrm{AD}$ decedents and mouse models. Here, we demonstrate that $A P O E 4$ and LRP1 influence $A \beta$ accumulation in an interactive manner in $\mathrm{AD}$ brains. Whereas apoE 4 facilitated $\mathrm{A} \beta$ deposition in mouse brains, this effect was reversed by neuronal LRP1 deletion. Our study provides molecular insight into how apoE4 exacerbates brain amyloid pathology during $\mathrm{AD}$ development.

\section{Results and Discussion}

Reduced brain LRP1 levels have been reported during aging and AD development (11). Positive regional associations between LRP1 and insoluble A 342 levels have also been detected in individuals without dementia (12) and in familial AD cases (13). To further address the contribution of LRP1 to A $\beta$ metabolism depending on APOE in $\mathrm{AD}$, we first examined the temporal cortices from pathologically con- 

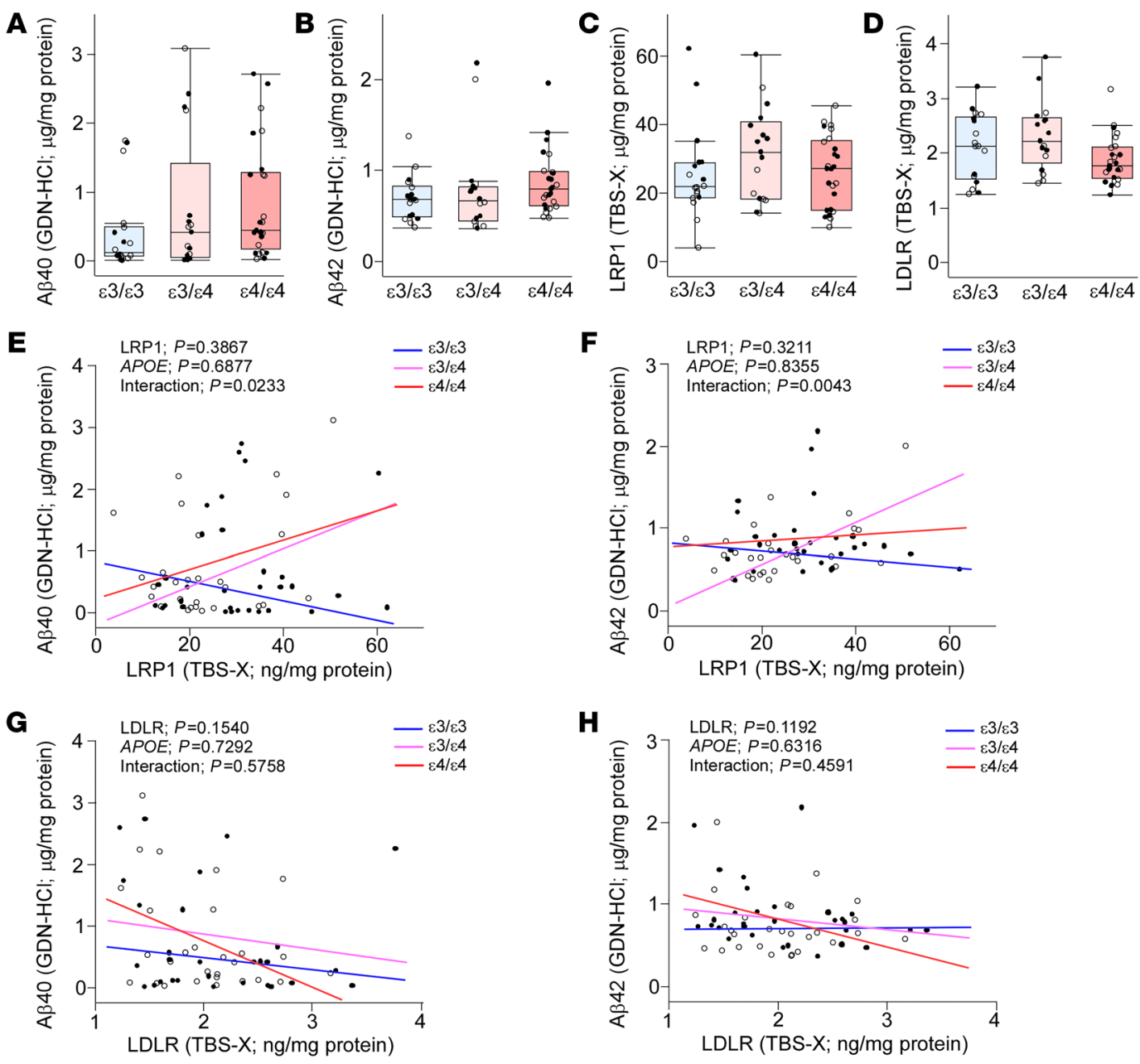

Figure 1. Impact of $A P O E 4$ on the association between levels of LRP1 and insoluble $A \beta$ in AD brains. (A-D) The concentrations of $A \beta 40$ (A) and $A \beta 42$ (B) in GDN-HCl fractions, and LRP1 (C) and LDLR (D) in TBS-X fractions of the temporal cortex samples are plotted as they relate to APOE genotype. Horizontal lines, boxes, and whiskers correspond to median, interquartile range (IQR), and the furthest points within $\times 1.5 \mathrm{IQR}$ from the box, respectively ( $n=17-25 / \mathrm{group}$ ). $(\mathbf{E}-\mathbf{H})$ The regression plots for concentrations between LRP1-A $\beta 40$ (E), LRP1-A $\beta 42$ (F), LDLR-A $\beta 40$ (C), and LDLR-A $\beta 42$ (H) are presented. Their interactions were assessed by ANCOVA by adjusting for age, sex, Braak stage, Thal phase, and averaged CAA scores. $R$ squares in each APOE genotype are as follows: (E) $\varepsilon 3 / \varepsilon 3, R^{2}=0.1165, P=0.1657 ; \varepsilon 3 / \varepsilon 4, R^{2}=0.1622, P=0.1090 ; \varepsilon 4 / \varepsilon 4, R^{2}=0.0843, P=0.1593 ;(F) \varepsilon 3 / \varepsilon 3, R^{2}=0.0725, P=0.2798 ; \varepsilon 3 / \varepsilon 4, R^{2}=0.3237, P=0.0214$; $\varepsilon 4 / \varepsilon 4, R^{2}=0.0111, P=0.6160 ;(\mathbf{C}) \varepsilon 3 / \varepsilon 3, R^{2}=0.0386, P=0.4500 ; \varepsilon 3 / \varepsilon 4, R^{2}=0.0228, P=0.5629 ; \varepsilon 4 / \varepsilon 4, R^{2}=0.1583, P=0.0489 ;$ and $(\mathbf{H}) \varepsilon 3 / \varepsilon 3, R^{2}=0.0005$, $P=0.9340 ; \varepsilon 3 / \varepsilon 4, R^{2}=0.0160, P=0.6404 ; \varepsilon 4 / \varepsilon 4, R^{2}=0.1857, P=0.0315$. White circles, data from female patients; black circles, data from male patients.

firmed late-onset AD patients with APOE $\varepsilon 3 / \varepsilon 3(n=18), \varepsilon 3 / \varepsilon 4(n=$ $17)$, or $\varepsilon 4 / \varepsilon 4(n=25)$ genotype through biochemical analyses (Supplemental Table 1; supplemental material available online with this article; https://doi.org/10.1172/JCI124853DS1). Brain tissues were lysed through a sequential extraction method based on differential solubility in TBS, TBS plus $1 \%$ Triton X-100 (TBS-X), and guanidine hydrochloride (GDN-HCl) (12-14). ELISA measurements of insoluble $\mathrm{A} \beta 40$ and $\mathrm{A} \beta 42$ in the GDN-HCl fraction, and LRP1 and LDL receptor (LDLR) in the TBS-X fraction, showed no significant differences among patients with different $A P O E$ genotypes after adjustment for age, sex, Braak stage (15), Thal phase (16), and cerebral amyloid angiopathy (CAA) severity (Figure 1, A-D). Furthermore, association of insoluble A $A$ levels with LRP1 and LDLR levels were evaluated using linear regression models depending on APOE genotype (Figure 1, E-H). Although negative correlations of the amounts of insoluble $A \beta 40$ or $A \beta 42$ with LRP1 levels were detected in AD patients with $A P O E \varepsilon 3 / \varepsilon 3$, they were positively associated in $\mathrm{AD}$ patients with $A P O E \varepsilon 3 / \varepsilon 4$ or $A P O E \varepsilon 4 / \varepsilon 4$ genotype. Analysis of covariance revealed a significant interaction effect of $A P O E$ genotype and LRP1 levels on insoluble $\mathrm{A} \beta 40(P=0.0233)$ or A $\beta 42$ level $(P=0.0043)$ after adjustment for age, sex, Braak stage, Thal phase, and CAA severity. There was no significant interaction between APOE 4 and LRP1 levels for soluble A $\beta$ (Supplemental Figure 1). While LDLR is another major apoE receptor in the brain, the $A P O E$ genotype did not influence the association between LDLR and insoluble A $\beta$ levels. These results imply that LRP1 is possibly involved in the molecular mechanisms of APOE4 exacerbation of brain $A \beta$ deposition in AD. Although CAA is often detected in AD brains, APOE genotype and/or LRP1 levels did not influence CAA severity in this cohort (Supplemental Figure 2).

Therefore, to investigate whether LRP1 mediates deleterious effects of APOE4 on A $\beta$ pathology, we generated neuron-specific LRP1-knockout mice ( $\mathrm{Lrp}^{1 / f / f}, \alpha \mathrm{CaMKII-Cre}{ }^{+}$; ; hereafter referred to as $n \mathrm{Lrp1}^{-/-}$mice) $(17,18)$ in the background of APP/PS1 amyloid model mice (APPswe/PSEN1 1 E9) (19), crossed with either APOE3-targeted 
A

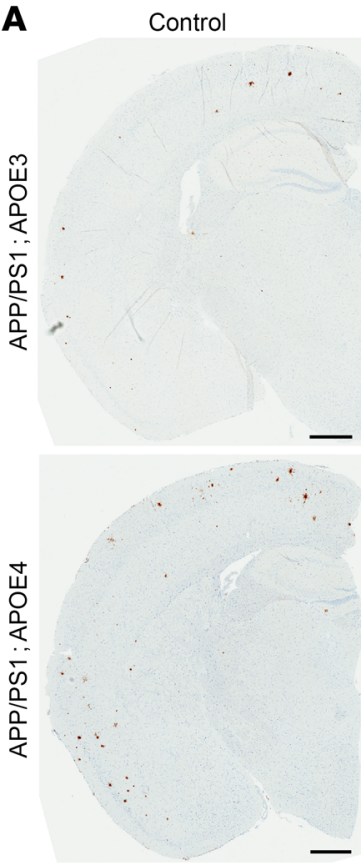

B

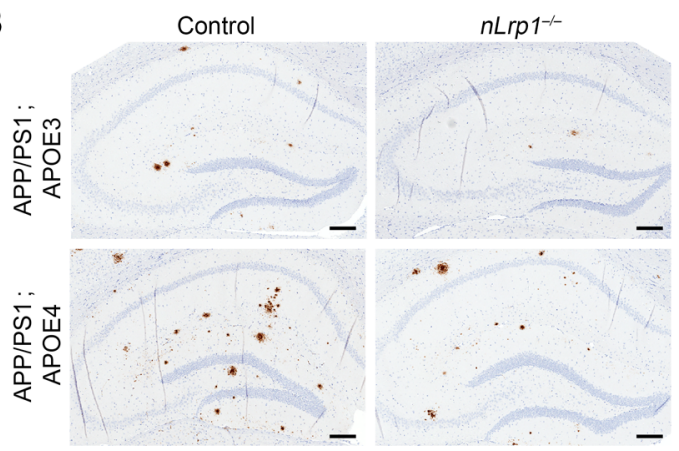

C

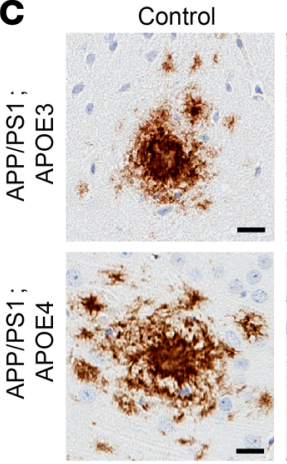

$n L r p 1^{-1-}$

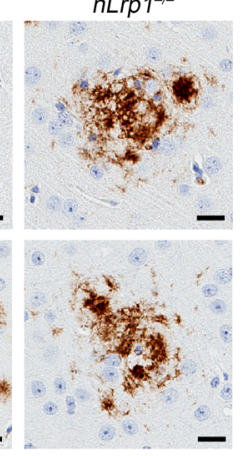

E
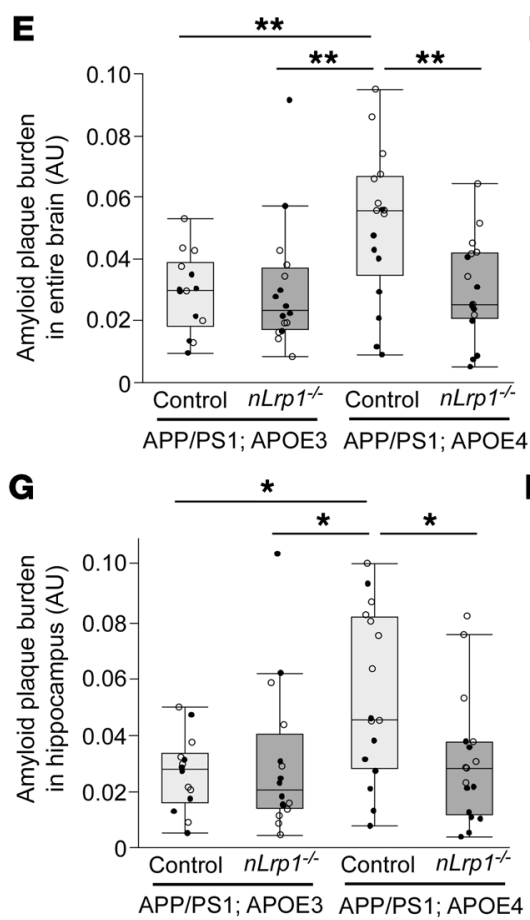
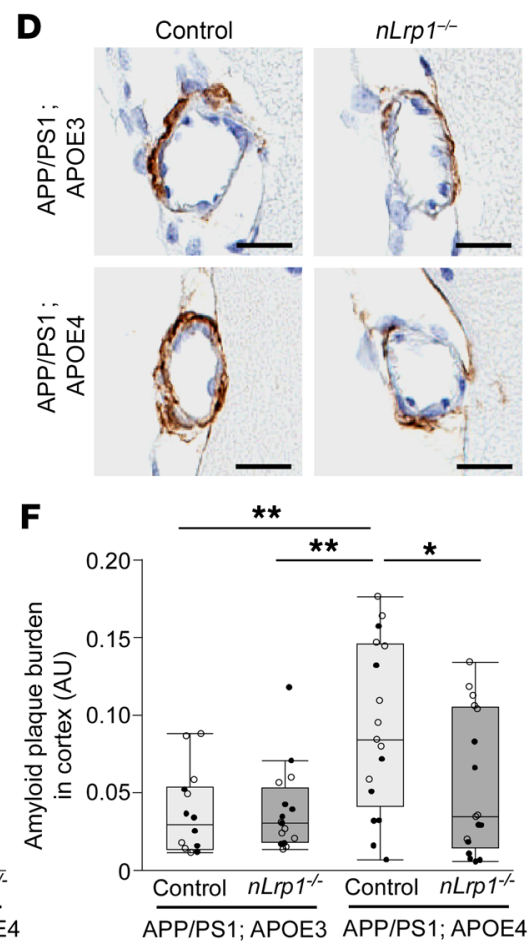

H

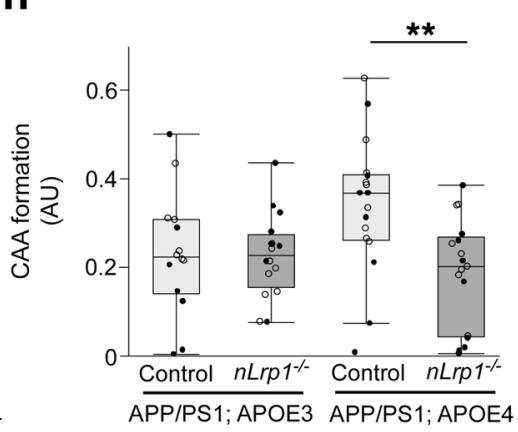

Figure 2. ApoE4 exacerbates brain amyloid plaque burden depending on neuronal LRP1 in APP/PS1 mice. (A-D) Brain A $\beta$ was stained in APP/PS1; APOE3 control, APP/PS1; APOE3; $n L r p 1^{-1-}$, APP/PS1; APOE4 control, and APP/PS1; APOE4; $n L r p 1^{-1-}$ mice with a pan-A $\beta$ antibody at 9 months of age. Representative images of entire brain (A), hippocampus (B), cortical amyloid plaque (C), and CAA in leptomeningeal arteries (D) are shown. Scale bars: $1 \mathrm{~mm}(\mathbf{A})$; $200 \mu \mathrm{m}$ (B); $20 \mu \mathrm{m}$ (C and D). (E-H) Amyloid plaque burdens in the entire brain (E), cortex (F) and hippocampus (G) and CAA formation in leptomeningeal arteries (H) from the mice were quantified through the Positive Pixel Count program (Aperio Technologies) ( $n=14-17 / g r o u p)$. Horizontal lines, boxes, and whiskers correspond to median, IQR, and the furthest points within $\times 1.5 \mathrm{IQR}$ from the box, respectively. ${ }^{*} P<0.05$; ${ }^{* *} P<0.01$, Tukey-Kramer post hoc analysis of 2-way ANOVA. White circles, data from female mice; black circles, data from male mice.

replacement (APOE3-TR) or APOE4-TR mice (20) (Supplemental Figure 3$)$. We first stained for $A \beta$ in brains from the following 4 groups of mice at the age of 9 months, when LRP1 is sufficiently deleted (17): (a) APP/PS1; APOE3 control; (b) APP/PS1; APOE3; $n L$ 1 $^{-1 /}$; (c) APP/

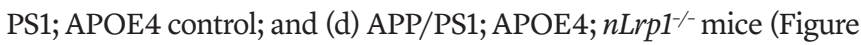
$2, A-D)$. Whereas $A \beta$ plaque burden was increased throughout the brain (Figure 2E), cortex (Figure 2F), and hippocampus (Figure 2G) in the presence of apoE4 compared with apoE3, apoE4's effect was diminished by neuronal LRP1 deficiency. Similarly, CAA formation was less in APP/PS1; APOE4; nLrp1/-- mice compared with APP/PS1; APOE 4 control mice (Figure $2 \mathrm{H}$ ). Brain $\mathrm{A} \beta$ immunoreactivity did not differ between mice in the APP/PS1; APOE3 control group and the $\mathrm{APP} / \mathrm{PS} 1$; APOE3; $n \mathrm{Lrp1}^{-/}$group. In addition, the amounts of $\mathrm{A} \beta 40$ (Figure 3, A-C) and A $\beta 42$ (Figure 3, D-F) in TBS-soluble fractions, detergent-soluble TBS-X fractions, or detergent-insoluble GDN-HCl fractions in the hippocampus from these mice were measured by ELI$\mathrm{SA}$ at 9 months of age. Consistent with the results from immunostaining, higher concentrations of insoluble $\mathrm{A} \beta 40$ (Figure $3 \mathrm{C}$ ) and $\mathrm{A} \beta 42$ (Figure $3 \mathrm{~F}$ ) in the $\mathrm{GDN}-\mathrm{HCl}$ fractions were detected in mice in the APP/PS1; APOE4 control group than in the APP/PS1; APOE3 con-

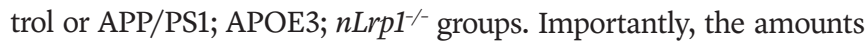
of insoluble $\mathrm{A} \beta$ in $\mathrm{APP} / \mathrm{PS} 1 ; \mathrm{APOE} 4 ; n L r p 1^{-/-}$mice were reduced to levels comparable to those of mice in the APP/PS1 group with apoE3. We did not detect significant effects of apoE4 and/or neuronal LRP1 deficiency on the levels of $\mathrm{A} \beta 40$ (Figure 3, $\mathrm{A}$ and $\mathrm{B}$ ) or $\mathrm{A} \beta 42$ (Figure $3, \mathrm{D}$ and $\mathrm{E}$ ) in the TBS or TBS-X fraction. Together, these results indicate that apoE4 predominantly affects $A \beta$ aggregation and deposition in mouse brains, which depend on a mechanism mediated by neu- 
A

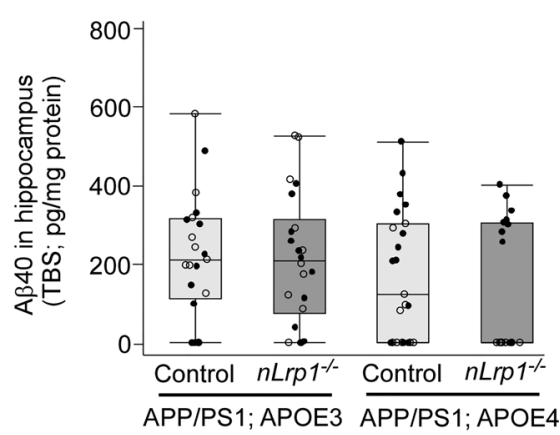

D

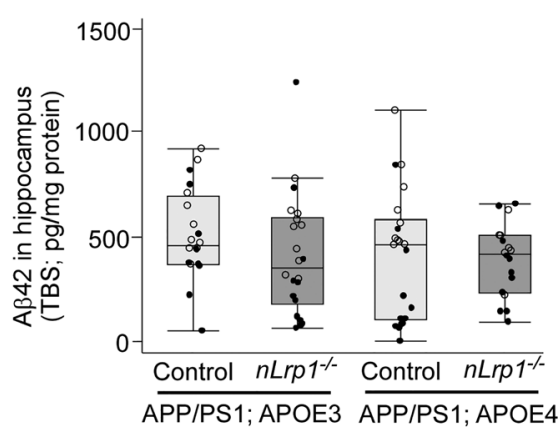

B

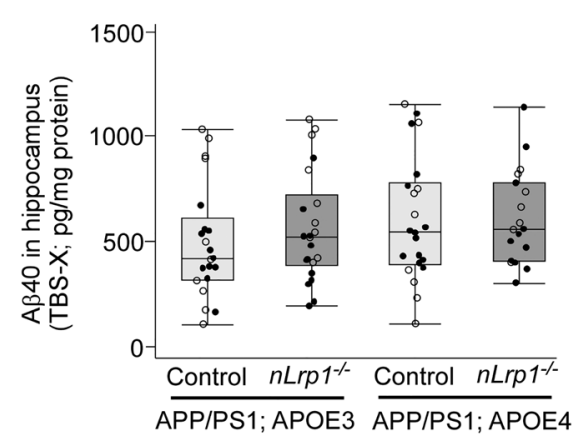

E

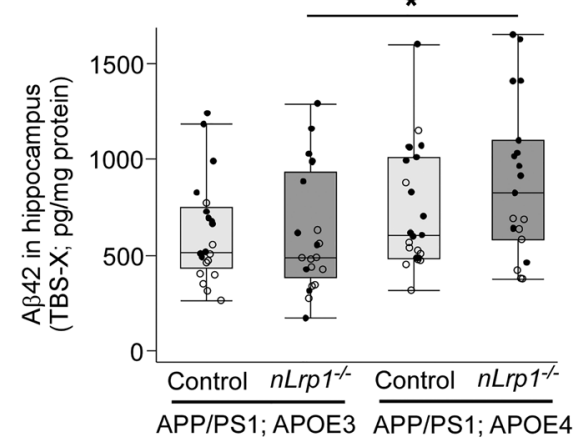

H

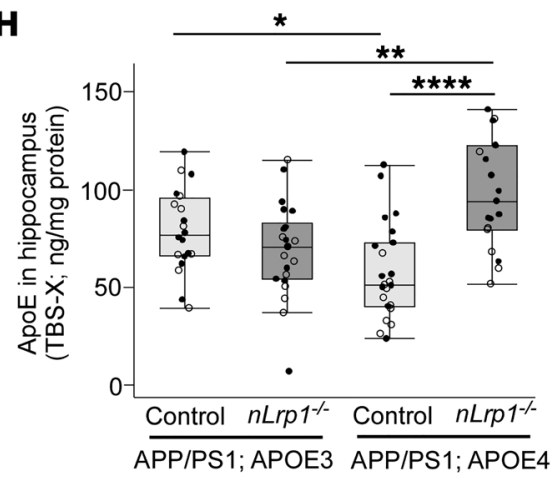

C

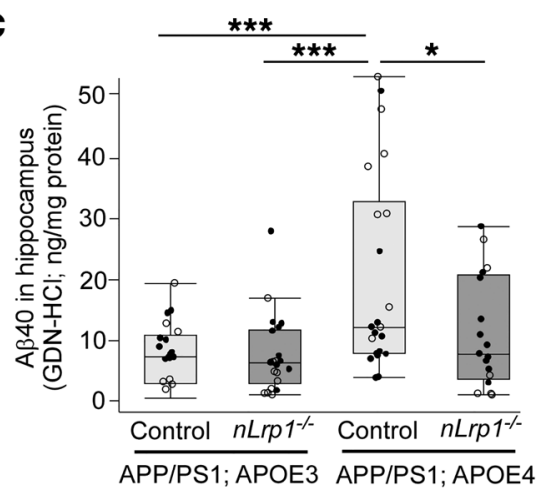

$\mathbf{F}$
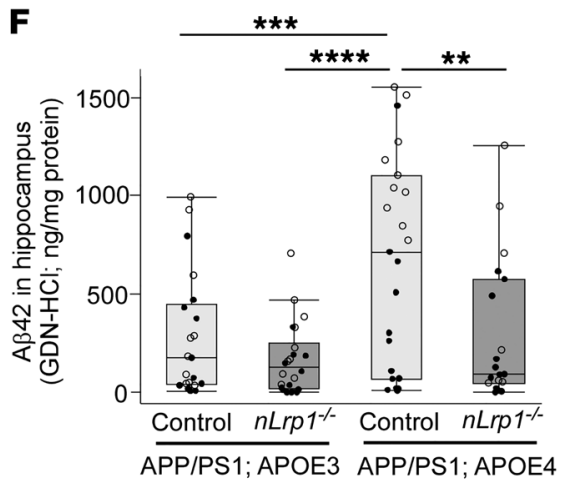

I

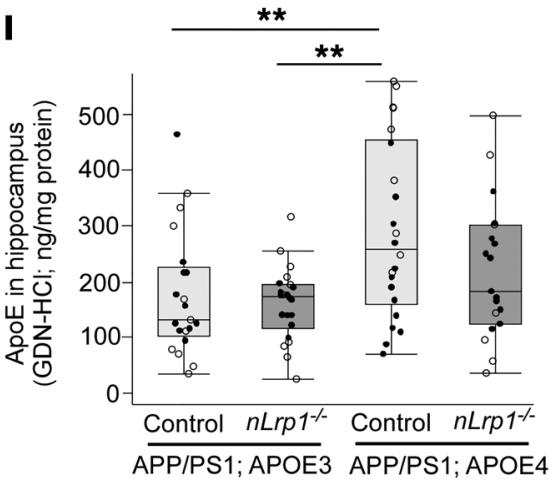

Figure 3. ApoE4 increases insoluble A $\beta$ levels in the hippocampus of APP/PS1 mice depending on neuronal LRP1. The concentrations of $A \beta 40$ (A-C), A 342 (D-F), and apoE (G-I) in the hippocampus extracted in TBS, TBS-X, and GDN-HCl from APP/PS1; APOE3 control, APP/PS1; APOE3; $n L$ rp1 ${ }^{-1-}$, APP/PS1;

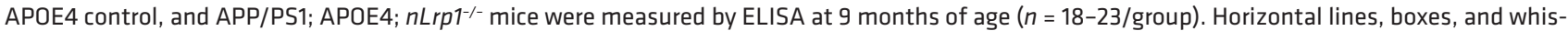
kers correspond to median, IQR, and the furthest points within $\times 1.5$ IQR from the box, respectively. ${ }^{*} P<0.05 ;{ }^{* *} P<0.01 ;{ }^{* *} P<0.001 ;{ }^{* * * *} P<0.0001$, Tukey-Kramer post hoc analysis of 2-way ANOVA. White circles, data from female mice; black circles, data from male mice.

ronal LRP1. Although apoE4 has been shown to increase the ratio of A $340 / A \beta 42$ in the Tg2576 amyloid model mice (21), no significant differences were observed in the ratios in our mouse models (Supplemental Figure 4, A-C). In addition, we did not detect any effects on APP C-terminal fragment $\beta$ (CTF $\beta$ ) levels among the mouse groups at 9 months of age (Supplemental Figure 4D), indicating that APP processing is not affected by apoE4 and/or neuronal LRP1 deficit. While glial cells play a substantial role in cellular clearance of $A \beta$, neither hippocampal astrocytes nor microglia from APP/PS1; APOE3 or APP/PS1; APOE4 mice (Supplemental Figure 5, A-D) were obviously affected by neuronal LRP1 deficiency (Supplemental Figure 5, A-D). In addition, there were no significant differences in hippocampal amounts of a postsynaptic marker, PSD95, and a presynaptic marker, synaptophysin (Supplemental Figure 5, E and F).
When hippocampal amounts of apoE were analyzed by ELISA in these mice (Figure 3G), TBS-soluble apoE levels were lower in the APP/PS1 mice with apoE4 than in those with apoE3, regardless of neuronal LRP1 deficiency. In the TBS-X fraction, which would be predicted to mainly contain membrane-associated proteins (22), APP/PS1; APOE4 control mice had lower APOE levels than APP/ PS1; APOE3 control mice. However, APP/PS1; APOE $4 ; n L r p 1^{-/}$mice possessed higher amounts of apoE in the TBS-X fraction than did APP/PS1; APOE4 control mice as well as APP/PS1 mice with apoE3 (Figure 3H). Insoluble apoE amounts in the $\mathrm{GDN}-\mathrm{HCl}$ fraction were higher in APP/PS1; APOE4 control mice than in APP/PS1; APOE3 control mice, which was not affected by neuronal LRP1 deficit (Figure 3I). These results imply that APOE4 is associated with reduced soluble apoE levels and increased insoluble apoE aggregates, where 


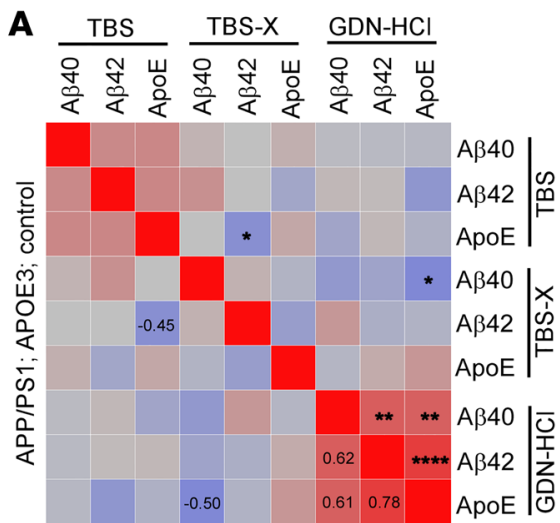

B
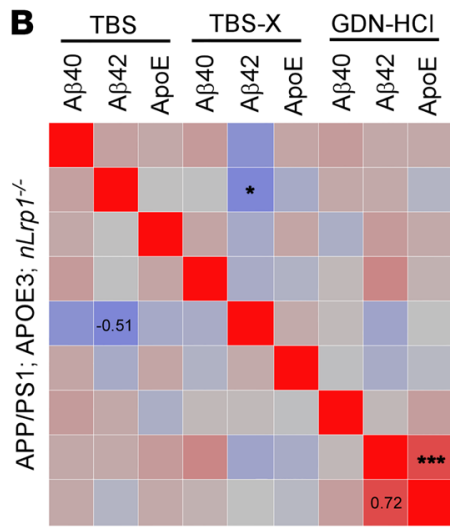

$A \beta 40 \mid \underset{\sim}{A}$

ApoE

A $\beta 40 \mid x$

A $\beta 42$ ç

ApoE

\begin{tabular}{l|l}
$A \beta 40$ & $\bar{O}$ \\
$A \beta 42$ & $I^{\prime}$ \\
ApoE & 0
\end{tabular}

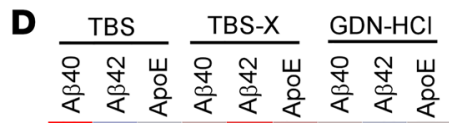

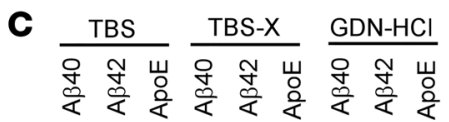

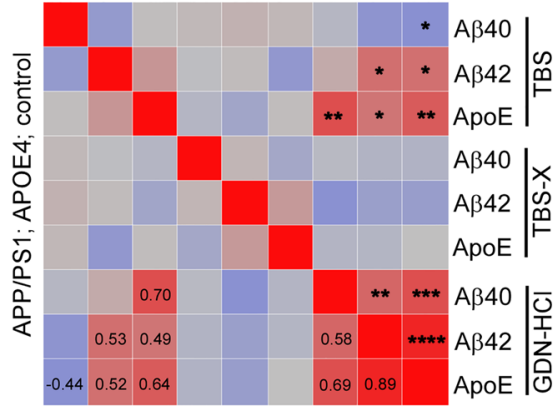

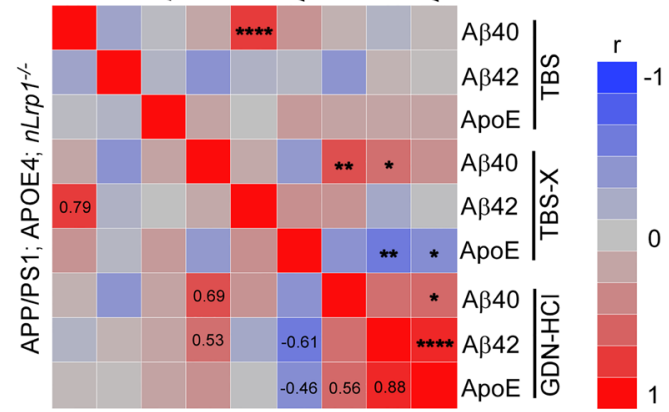

Figure 4. Influences of apoE4 and neuronal LRP1 deficiency on the correlations among $A \beta$ and apoE in the hippocampus of APP/PS1 mice. Heatmaps of Spearman's rank correlation coefficients summarizing the correlations among hippocampus levels of $A \beta 40, A \beta 42$, and apoE are shown. These data are from measurements by ELISA in TBS, TBS-X, and GDN-HCl fractions of APP/PS1; APOE3 control (A), APP/PS1; APOE3; $n L r p 1^{-1-}$ (B), APP/PS1; APOE4 control mice (C), and APP/PS1; APOE4; $n L r \mathrm{pr}^{-/-}$(D) mice at 9 months of age ( $n$ $=18-23$ /group). ${ }^{*} P<0.05 ;{ }^{* *} P<0.01$; ${ }^{* * *} P<0.001 ;{ }^{* * *} P<0.0001$, Pearson's product-moment correlation.
LRP1 may mediate the trafficking of membrane-associated apoE4 in neurons. In addition, we also analyzed the correlations among hippocampal A $340, A \beta 42$, and apoE levels in TBS, TBS-X, and GDN-HCl fractions among the 4 groups of mice (Figure $4, \mathrm{~A}-\mathrm{D}$ ). While insoluble levels of $A \beta 40, A \beta 42$, and apoE were strongly correlated with each other in both APP/PS1; APOE3 control and APP/PS1; apoE4 control mice, the interaction between insoluble $A \beta 40$ and $A \beta 42$ was weakened in the absence of neuronal LRP1. Furthermore, apoE levels in the TBS-X fraction displayed significant inverse correlations with insoluble $\mathrm{A} \beta 42$ and apoE levels in the GDN-HCl fraction in $\mathrm{APP} / \mathrm{PS} 1$; APOE $4 ; n \mathrm{Lrp1}^{-/-}$mice $(P=0.0058)$. Since A $\beta$ deposition was reduced in $\mathrm{APP} / \mathrm{PS} 1 ; \mathrm{APOE} 4 ; n L r p 1^{-/-}$mice compared with APP/ PS1; APOE4 control mice, these results suggest that neuronal LRP1 deficiency preserves more apoE4 in the detergent soluble fraction, likely on the cell surface, resulting in prevention of APOE4-related $\mathrm{A} \beta$ aggregation. Indeed, apoE3 or apoE 4 competes with $\mathrm{A} \beta$ for the association with cell-surface HSPGs (23). More importantly, neuronal heparan sulphate deficiency substantially suppresses $A \beta$ plaque formation in APP/PS1 mice (24). Therefore, future studies should clarify how HSPGs or other apoE receptors contribute to apoE4- and neuronal LRP1-mediated A $\beta$ metabolism during AD.

Whereas clearance of soluble $A \beta$ in the interstitial fluid (ISF) was directly measured by in vivo microdialysis in the hippocampus of mice in the APP/PS1; APOE3 control, APP/PS1; APOE3; $n \mathrm{Lrp1}^{-/-}$, $\mathrm{APP} / \mathrm{PS} 1 ; \mathrm{APOE} 4$ control, and APP/PS1; APOE $4 ; n L r p 1^{-/-}$groups at the age of 12-14 months, we did not observe an effect of neuronal LRP1 deficiency on ISF A $\beta$ clearance in the mice with apoE3 or apoE4, although the presence of amyloid pathology at this age may complicate the determination of clearance rate due to buffering of soluble $A \beta$ through incorporation into plaques (Supplemental Figure 6). We previously demonstrated that cortical $A \beta$ clearance was disturbed in APP/PS1; $n L_{r p 1^{-/}}$mice with a murine Apoe background compared with control APP/PS1 mice, thereby exacerbating $A \beta$ pathology (18). Since murine apoE is shown to coaggregate with A $\beta$ more robustly than human apoE (25), neuronal LRP1 may mediate endocytosis of the complex of murine apoE and $A \beta$ in mouse brains, rather than free $A \beta$. Furthermore, as human apoE likely antagonizes LRP1 for cellular $A \beta$ uptake (26), the effect of neuronal LRP1 deficiency on ISF A $\beta$ clearance may not be evident in the presence of apoE3 or apoE4 compared with the condition with murine apoE alone. Although further studies are needed, it is also possible that neuronal LRP1 predominantly contributes to the apoE4-related $A \beta$ aggregation process rather than soluble $A \beta$ clearance. In addition, unlike the results of those from the hippocampus, the deletion of neuronal LRP1 did not influence the concentrations of $\mathrm{A} \beta$ and apoE in the cortex of APP/PS1 mice expressing apoE3 or apoE4 (Supplemental Figure 7). Thus, our findings indicate that neuronal LRP1 function in A $\beta$ metabolism differs depending on the presence of murine and human apoE or brain regions. It may be important to consider potential effects of these factors whenever investigating roles of apoE-related molecules in $\mathrm{A} \beta$ metabolism using mouse models. In summary, our study has shown that apoE4 aggravates $A \beta$ pathology by interacting with neuronal LRP1 in mouse models, which supports our observation that APOE4 facilitates the positive association between LRP1 and insoluble A $\beta$ levels in postmortem AD brains. Recent findings have demonstrated that increasing apoE 4 during the $A \beta$-seeding phase (0-6 months of age) in APP/PS1 mice specifically leads to exacerbated amyloid 
pathology (9). Thus, it is predicted that neuronal LRP1 deficiency also prevents apoE4-related $A \beta$ aggregation at an early stage. One limitation of our study is that we could not analyze $A \beta$ pathology in older mice due to a reduced survival rate at the age of 12 months, for unknown reasons. At older ages, neuronal LRP1 deficiency may accelerate $A \beta$ deposition independently of apoE4. In this regard, suppressing LRP1 levels may not be a suitable approach as potential AD therapy because LRP1 plays a critical role in maintaining brain homeostasis $(5,10)$. Nonetheless, increasing apoE4 amounts in the TBS-X-soluble fraction may be an alternative therapeutic intervention for $\mathrm{AD}$ with APOE4. Modifying apoE4 solubility and/ or retaining more apoE4 onto the cell surface at an early stage of AD could potentially be beneficial, in addition to lowering the amount of apoE4 aggregates through treatment with specific antisense oligonucleotides (27) or antibodies (28). Taken together, our results indicate that exploring interactive roles of apoE and apoE receptors in $\mathrm{A} \beta$ metabolism would help us to better understand the mechanisms underlying the contribution of APOE4 to the risk of $\mathrm{AD}$ development and progression.

\section{Methods}

Study approval. The Mayo Clinic Institutional Review Board approved all protocols for human study in which experimental procedures were conducted. All subjects gave informed consent. The Mayo Clinic Institutional Animal Care and Use Committee approved all animal procedures, which were conducted in accordance with the regulations of the American Association for the Accreditation of Laboratory Animal Care.

Details about experimental procedures are provided in the Supplemental Methods.

\section{Author contributions}

MT, MLH, GB, and TK designed the research studies. MT, MLH, CCL, MS, TA, HO, YY, YAM, MEM, PMS, KW, SG, and DWD conducted experiments and acquired data. MT, MLH, CCL, MS, TA, HO, YY, YAM, MEM, PMS, KW, SG, DWD, GB, and TK analyzed the data. MT, MLH, GB, and TK wrote the first draft. All authors contributed to writing the final manuscript.

\section{Acknowledgments}

This work was supported by NIH grants (R37AG027924, P01NS074969, R01AG035355, and RF1AG051504 to GB; R21AG052423 to TK) and an Alzheimer's Association New Investigator Research Grant, 2014NIRG304190 (to TK).

Address correspondence to: Takahisa Kanekiyo or Guojun $\mathrm{Bu}$, Department of Neuroscience, Mayo Clinic Jacksonville, 4500 San Pablo Road, Jacksonville, FL 32224, USA. Phone: 904.953.1064; E-mail:kanekiyo.takahisa@mayo.edu(TK).Phone:904.786.3419; E-mail:bu.guojun@mayo.edu (GB).
1. [No authors listed]. 2018 Alzheimer's disease facts figures. Alzheimer's \& Dementia. 2018;14(3):367-429.

2. Blennow K, de Leon MJ, Zetterberg H. Alzheimer's disease. Lancet. 2006;368(9533):387-403.

3. Selkoe DJ. Deciphering the genesis and fate of amyloid beta-protein yields novel therapies for Alzheimer disease. JClin Invest. 2002;110(10):1375-1381.

4. Karran E, Mercken M, De Strooper B. The amyloid cascade hypothesis for Alzheimer's disease: an appraisal for the development of therapeutics. Nat Rev Drug Discov. 2011;10(9):698-712.

5. Bu G. Apolipoprotein E and its receptors in Alzheimer's disease: pathways, pathogenesis and therapy. Nat Rev Neurosci. 2009;10(5):333-344.

6. Corder EH, et al. Gene dose of apolipoprotein $\mathrm{E}$ type 4 allele and the risk of Alzheimer's disease in late onset families. Science. 1993;261(5123):921-923.

7. Kanekiyo T, Xu H, Bu G. ApoE and Aß in Alzheimer's disease: accidental encounters or partners? Neuron. 2014;81(4):740-754.

8. Castellano JM, et al. Human apoE isoforms differentially regulate brain amyloid- $\beta$ peptide clearance. Sci Transl Med. 2011;3(89):89ra57.

9. Liu CC, et al. ApoE4 accelerates early seeding of amyloid pathology. Neuron. 2017;96(5):1024-1032.e3.

10. Kanekiyo T, Bu G. The low-density lipoprotein receptor-related protein 1 and amyloid- $\beta$ clearance in Alzheimer's disease. Front Aging Neurosci. 2014;6:93.

11. Kang DE, et al. Modulation of amyloid beta-protein clearance and Alzheimer's disease susceptibility by the LDL receptor-related protein pathway. J Clin Invest. 2000;106(9):1159-1166.

12. Shinohara M, Petersen RC, Dickson DW, Bu G. Brain regional correlation of amyloid- $\beta$ with synapses and apolipoprotein $\mathrm{E}$ in non-demented individuals: potential mechanisms underlying regional vulnerability to amyloid- $\beta$ accumulation. Acta Neuropathol. 2013;125(4):535-547.

13. Shinohara M, et al. Regional distribution of synaptic markers and APP correlate with distinct clinicopathological features in sporadic and familial Alzheimer's disease. Brain. 2014;137(Pt 5):1533-1549.

14. Shinohara M, et al. Impact of sex and APOE4 on cerebral amyloid angiopathy in Alzheimer's disease. Acta Neuropathol. 2016;132(2):225-234.

15. Braak H, Braak E. Neuropathological stageing of Alzheimer-related changes. Acta Neuropathol. 1991;82(4):239-259.

16. Thal DR, Rüb U, Orantes M, Braak H. Phases of A beta-deposition in the human brain and its relevance for the development of AD. Neurology. 2002;58(12):1791-1800.

17. Liu Q, et al. Neuronal LRP1 knockout in adult mice leads to impaired brain lipid metabolism and progressive, age-dependent synapse loss and neurodegeneration. J Neurosci. 2010;30(50):17068-17078.

18. Kanekiyo T, et al. Neuronal clearance of amyloid- $\beta$ by endocytic receptor LRP1. J Neurosci. 2013;33(49):19276-19283.

19. Borchelt DR, et al. Accelerated amyloid deposition in the brains of transgenic mice coexpressing mutant presenilin 1 and amyloid precursor proteins. Neuron. 1997;19(4):939-945.

20. Knouff C, et al. Apo E structure determines VLDL clearance and atherosclerosis risk in mice. JClin Invest. 1999;103(11):1579-1586.

21. Fryer JD, et al. Human apolipoprotein E4 alters the amyloid-beta 40:42 ratio and promotes the formation of cerebral amyloid angiopathy in an amyloid precursor protein transgenic model. J Neurosci. 2005;25(11):2803-2810.

22 . Youmans KL, et al. Amyloid- $\beta 42$ alters apolipoprotein E solubility in brains of mice with five familial AD mutations. J Neurosci Methods. 2011;196(1):51-59.

23. Fu Y, et al. Apolipoprotein E lipoprotein particles inhibit amyloid- $\beta$ uptake through cell surface heparan sulphate proteoglycan. Mol Neurodegener. 2016;11(1):37.

24. Liu CC, et al. Neuronal heparan sulfates promote amyloid pathology by modulating brain amyloid- $\beta$ clearance and aggregation in Alzheimer's disease. Sci Transl Med. 2016;8(332):332ra44.

25. Liao F, et al. Murine versus human apolipoprotein E4: differential facilitation of and co-localization in cerebral amyloid angiopathy and amyloid plaques in APP transgenic mouse models. Acta Neuropathol Commun. 2015;3:70.

26. Verghese PB, et al. ApoE influences amyloid- $\beta$ $(\mathrm{A} \beta)$ clearance despite minimal apoE/A $\beta$ association in physiological conditions. Proc Natl Acad Sci U S A. 2013;110(19):E1807-E1816.

27. Huynh TV, et al. Age-dependent effects of apoE reduction using antisense oligonucleotides in a model of $\beta$-amyloidosis. Neuron. 2017;96(5):1013-1023.e4.

28. Liao F, et al. Targeting of nonlipidated, aggregated apoE with antibodies inhibits amyloid accumulation. JClin Invest. 2018;128(5):2144-2155. 